\title{
(9) Comparison of CT versus MRI measurements of transverse atlantal ligament integrity in craniovertebral junction injuries. Part 2: A new CT-based alternative for assessing transverse ligament integrity
}

\author{
Luis Perez-Orribo, MD, PhD, ${ }^{1}$ Samuel Kalb, MD, ${ }^{2}$ Laura A. Snyder, MD, ${ }^{2}$ Forrest Hsu, MD, ${ }^{2}$ \\ Devika Malhotra, MD, ${ }^{1}$ Richard D. Lefevre, BS, ${ }^{1}$ Ali M. Elhadi, MD, ${ }^{2}$ Anna G. U. S. Newcomb, MS, ${ }^{1}$ \\ Nicholas Theodore, MD, ${ }^{2}$ and Neil R. Crawford, $\mathrm{PhD}^{1}$ \\ ${ }^{1}$ Spinal Biomechanics Laboratory and 2Department of Neurosurgery, Barrow Neurological Institute, St. Joseph's Hospital and \\ Medical Center, Phoenix, Arizona
}

\begin{abstract}
OBJECTIVE The rule of Spence is inaccurate for assessing integrity of the transverse atlantal ligament (TAL). Because $\mathrm{CT}$ is quick and easy to perform at most trauma centers, the authors propose a novel sequence of obtaining $2 \mathrm{CT}$ scans to improve the diagnosis of TAL impairment. The sensitivity of a new CT-based method for diagnosing a TAL injury in a cadaveric model was assessed.

METHODS Ten human cadaveric occipitocervical specimens were mounted horizontally in a supine posture with wooden inserts attached to the back of the skull to maintain a neutral or flexed $\left(10^{\circ}\right)$ posture. Specimens were scanned in neutral and flexed postures in a total of 4 conditions ( 3 conditions in each specimen): 1$)$ intact $(n=10)$; either $2 A)$ after a simulated Jefferson fracture with an intact TAL $(n=5)$ or $2 B$ ) after a TAL disruption with no Jefferson fracture $(n=5)$; and 3) after TAL disruption and a simulated Jefferson fracture $(n=10)$. The atlantodental interval $(A D I)$ and cross-sectional canal area were measured.
\end{abstract}

RESULTS From the neutral to the flexed posture, ADI increased an average of $2.5 \%$ in intact spines, $6.25 \%$ after a Jefferson fracture without TAL disruption, 34\% after a TAL disruption without fracture, and 25\% after TAL disruption with fracture. The increase in ADI was significant with both TAL disruption and TAL disruption and fracture $(p<0.005)$ but not in the other 2 conditions ( $p>0.6)$. Changes in spinal canal area were not significant $(p>0.70)$.

CONCLUSIONS This novel method was more sensitive than the rule of Spence for evaluating the integrity of the TAL on $\mathrm{CT}$ and does not increase the risk of further neurological damage.

http://thejns.org/doi/abs/10.3171/2015.9.SPINE13807

KEY WORDS transverse atlantal ligament; atlas fracture; measurement; axis fracture; atlantodental interval; atlantoaxial overhang; trauma

$\mathrm{T}$ HE C-1 vertebra is a ring structure with 2 lateral masses bridged anteriorly and posteriorly by 2 arches. The cohesion of the craniovertebral junction $(\mathrm{CVJ})$ results from the ligaments of the facet capsules (occiput-C1 and $\mathrm{C} 1-2)$, anterior longitudinal ligament, tectorial membrane, apical and alar ligaments, and cruciate ligament formed by the transverse atlantal ligament (TAL) and longitudinal ligament. ${ }^{9,13}$ The medial tubercles of the lateral masses provide points of attachment for the TAL on each side (Fig. 1). Posteriorly, the myoligamentous complex helps stabilize the CVJ. ${ }^{23,25}$

As the first cervical vertebra, the atlas has a crucial function in the anatomy of the upper cervical spine, acting as a transitional structure between the occiput and cervical spine. Because of the atlas' ring shape and the lack of intervertebral disc, the atlantoaxial joint has the greatest range of motion and flexibility of all levels of the spinal column. With such flexibility, traumatic force vectors pro- 


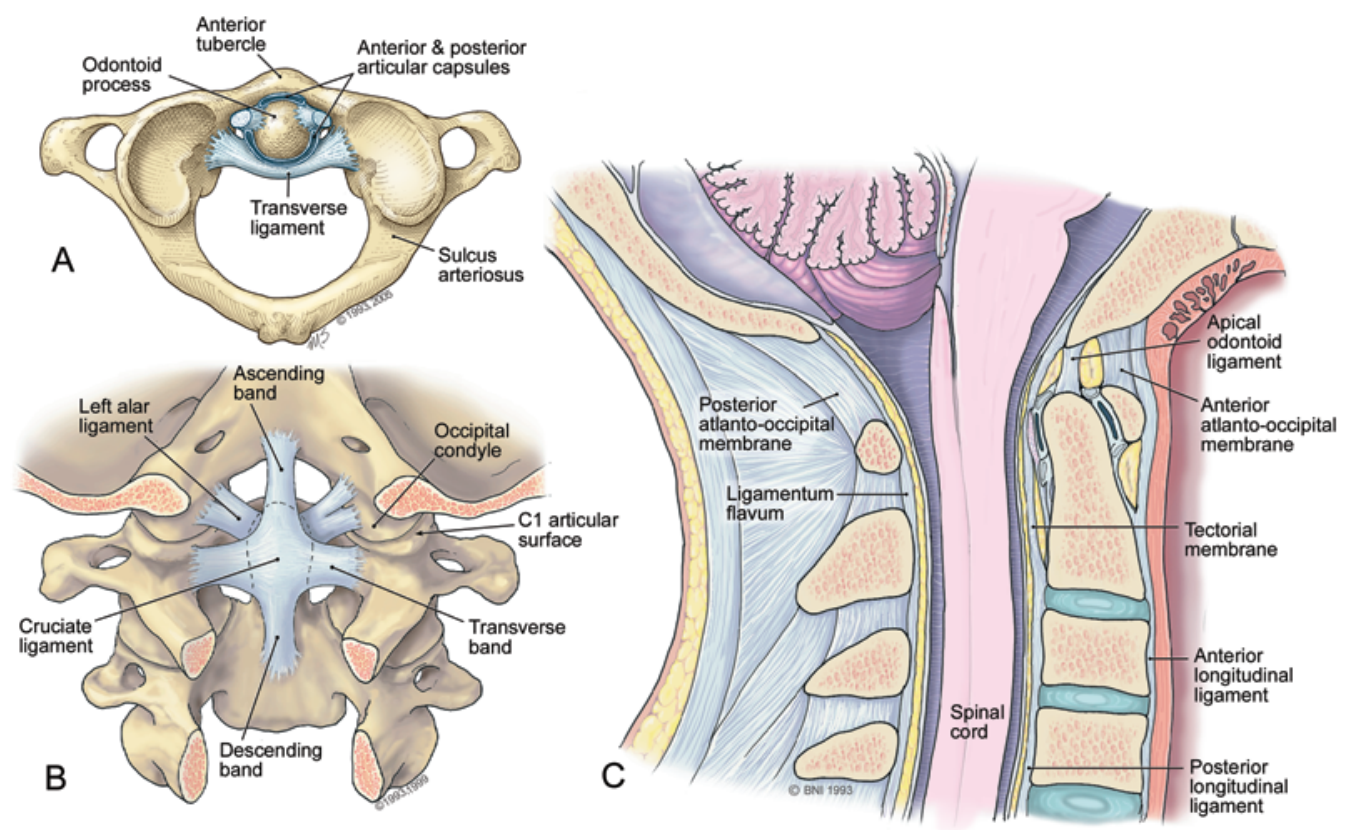

FIG. 1. A: Superior view of the atlas showing the attachments of the TAL to the medial tubercles and atlantodental articular joint. B: Posterior view without the tectorial membrane showing the cruciate ligament complex along with the alar ligaments attaching the dens to the occipital condyles. The Oc-C1 and C1-2 capsules (not shown here) are also crucial for the stability of the CVJ. C: Sagittal view demonstrating the posterior longitudinal ligament continuing as the tectorial membrane with insertion into the clivus. Also shown is the apical ligament, which attaches to the dens and basion and was obscured by the ascending band of cruciate ligament in panel B. From Dickman CA, Crawford NR, Brantley AGU, Sonntag VKH, Koeneman JB: In vitro cervical spine biomechanical testing. BNI Quarterly 9:17-26, 1993. Copyright Barrow Neurological Institute, Phoenix, Arizona. Published with permission.

duce unique and complicated fracture patterns that require special care for their diagnosis and treatment. ${ }^{9}$

Fractures of the C-1 vertebra account for $25 \%$ of craniovertebral injuries, $3 \%-13 \%$ of cervical spine injuries, and $1.3 \%-2 \%$ of all spinal injuries. ${ }^{7}$ Atlas fractures are often associated with other injuries of the cervical spine. Axis fractures are associated with $40 \%-44 \%$ of atlas fractures and also with other soft-tissue injuries such as the rupture of the TAL?

In terms of the capacity of atlas fractures to heal, the status of the TAL is a critical factor because it plays a crucial role in determining atlantoaxial stability by maintaining the odontoid process against the anterior arch of the atlas. ${ }^{17}$ Atlas fractures are defined as stable or unstable on the basis of the inferred integrity of the TAL. According to Dickman et al., ${ }^{2}$ all Type I TAL fractures and most Type IIB fractures require surgical stabilization to heal correctly and to avoid future instability and the risk of fatal neural damage. Consequently, an early diagnosis of TAL impairment is essential.

Spence et al. ${ }^{20}$ proposed that the lateral spread of the atlantal lateral masses with respect to the lateral limits of $\mathrm{C}-2$ as measured on a transoral radiograph could be used to infer impairment of the TAL. Their criterion for suspecting a ruptured TAL was a spread greater than or equal to $7 \mathrm{~mm}$. However, about $61 \%$ of TAL injuries are missed when this criterion is used..$^{2,16}$ Another measurement that is widely used to assess the integrity of the TAL is the distance between the anterior arch of C-1 and the odontoid process, known as the atlantodental interval (ADI), as measured on flexion-extension radiographs. A normal ADI is defined as $3 \mathrm{~mm}$ or less. Approximately $25 \%$ of patients with an impairment of the TAL are considered to have normal anatomy using this criterion. ${ }^{2}$ However, unobstructed true lateral radiographs for accurately assessing ADI are difficult to obtain.

In a previous study, we assessed patients with traumatic injuries of the CVJ, using CT as an alternative to MRI to evaluate the integrity of the TAL. ${ }^{18}$ The objective of the current study was to evaluate a new method for diagnosing TAL disruption based on measurements from conventional CT scans of cadaveric specimens held in a neutral posture followed by slightly flexed postures. CT scanning was used because it is the most widely used modality for diagnosing injuries of the $\mathrm{CVJ}$ and because it is more commonly available in emergency departments, compared with the less accessible and more expensive MRI.

\section{Methods \\ Cadaveric Specimens}

The CVJ (occiput-C2 ) of 10 cadaveric specimens with a skull base and an intact portion of the posterior skull were used. Five specimens were from male donors and 5 were from female donors (mean age 52 years, age range 30-60 years). All specimens were obtained fresh frozen and unembalmed. Because cadaveric muscle tissue would add excessive resistance to movement, all muscle and passive soft tissue were dissected carefully without damaging the ligaments, discs, or joint capsules. The bone mineral 
density of all specimens was measured using anteroposterior dual-energy x-ray absorptiometry to ensure that no specimen had poor bone quality.

Metallic artifacts on CT were was avoided by using plastic household cable ties to secure specimens to custom-made vertically oriented flat wooden boards. Cable ties were passed through both vertebral foramina of C-2 and through holes in the board to secure the caudal surface of C-2 rigidly to the board. A 2-cm-diameter wooden dowel was fixed to the skull base through a hole drilled at the level of the torcular herophili. In each specimen, the dowel length was adjusted so that the dowel rested against the board with the CVJ aligned in a neutral position (Fig. 2 upper). Five such preparations of vertically oriented boards with attached CVJs were mounted to a single horizontal board to allow the 5 specimens to be scanned together. Furthermore, wooden wedges were fashioned to create a $10^{\circ}$ flexion angle when inserted between the vertically oriented board and dowel (Fig. 2 lower). A flexion angle of $10^{\circ}$ was selected based on a rough approximation of how much flexion might be achieved when a pillow is placed behind a patient's head. To ensure consistent angulation, we sized wedges for each specimen so that the vertex was positioned at approximately the center of rotation of $\mathrm{Oc}-\mathrm{C} 1-\mathrm{C} 2$ during flexion-extension.

\section{Quantitative Anatomical Analysis}

In the intact condition, specimens were scanned twice, once in neutral posture and once with wedges inserted to induce slight flexion $\left(10^{\circ}\right)$. We obtained 3D CT scans using $0.625-\mathrm{mm}$ axial slice resolution in a clinical CT scanner (LightSpeed, GE Systems).

For each specimen and in both postures, linear measurements were obtained from the CT scans by using clinical image management software (Dominator, DR Systems, Inc.). Linear measurements included ADI and the external anteroposterior and lateral dimensions of the atlas. The area of the spinal canal at the midheight of C-1 was measured in neutral and flexed postures.

To obtain calibrated and correctly oriented 2D images for area measurements, we used custom software to insert a graphic object of known length on the display and to rotate the resliced $\mathrm{CT}$ volume until $\mathrm{C}-1$ was aligned with the image plane (Fig. 3). Image analysis software (Image Tool, UTHSC) was then used to outline the spinal canal on this image slice and to quantify the area of the spinal canal.

After CT scans were obtained in the intact condition, a typical Jefferson fracture (Type III of Jefferson's classification and Type II of Landells and Van Peteghem's classification), ${ }^{2,13}$ was simulated in 5 of the specimens by cutting bilaterally the anterior and posterior arches of the atlas at their union to the lateral masses (Group A). These cuts were made using a high-speed drill (Dremel Corp.) using a small (1-mm) fluted bit. Drilling was performed meticulously to respect the entire CVJ-ligamentous complex.

After CT scans were obtained in the remaining 5 intact specimens (Group B), the TAL was sectioned to reproduce a Dickman et al. ${ }^{2}$ Type I TAL injury, which always requires surgical treatment. The TAL was completely transected unilaterally by cutting the ligament at its anatomi-

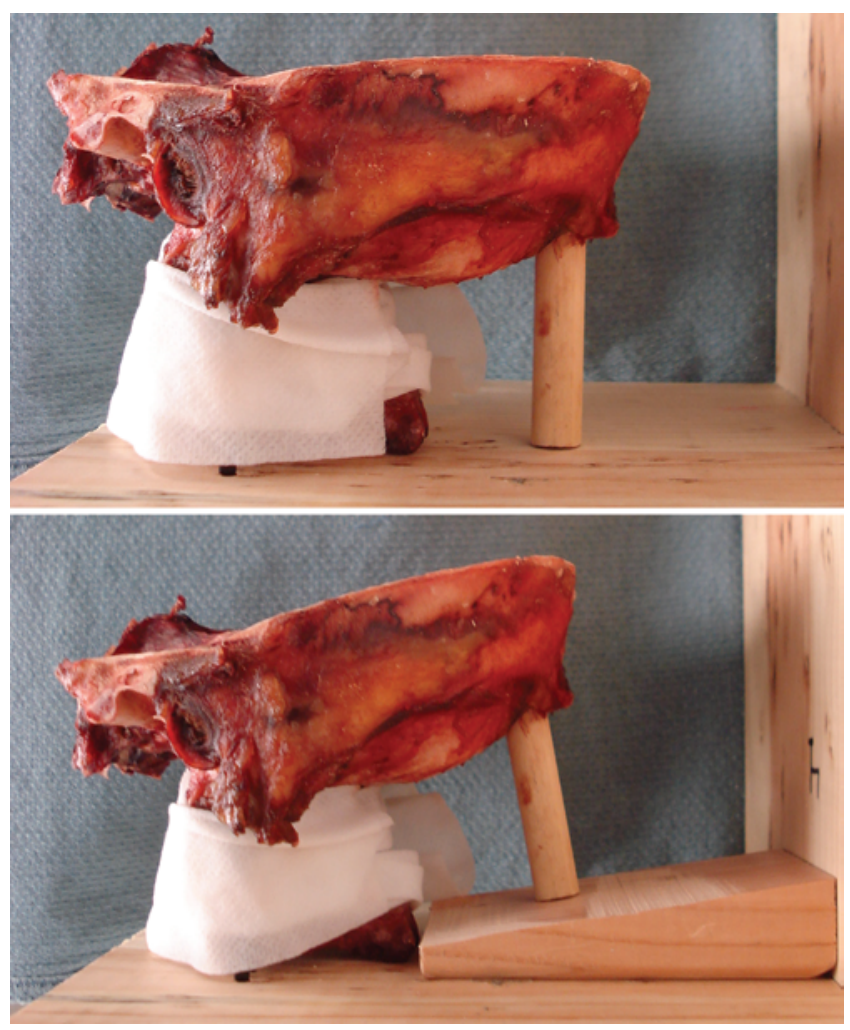

FIG. 2. Upper: Human CVJ carefully dissected and fixed in a wood holder using plastic ties inserted through the vertebral foramina of C-2. In all specimens, a wood cylinder was placed at the level of the torcular herophili to obtain a neutral position. Lower: Wood wedge used to create slight flexion $\left(10^{\circ}\right)$ of the CVJ.

cal insertion into the left or right atlantal medial tubercle. CT scans of these specimens were then obtained in neutral and slightly flexed $\left(10^{\circ}\right)$ postures as described above.

After the fractures and the TAL injuries were created, all 10 specimens (Groups A and B) were briefly compressed axially using a deadweight force of $135 \mathrm{~N}$. This step was intended to reproduce the typical axial load that would be present from muscle forces and gravity after this kind of injury and to ensure that any bone fragments bridging between the atlas sections were dislodged. The specimens were then rescanned in the neutral and flexed positions, and measurements were obtained in the same fashion as in the first step.

Finally, further injury was induced in all 10 specimens to merge the 2 groups into a single group with an identical final condition resulting from 2 different injury sequences. That is, the TAL was sectioned or a Jefferson fracture was simulated initially, followed by the second injury, such that all specimens had both a Jefferson fracture and disruption of the TAL. CT scans of all 10 specimens were obtained again in neutral and flexed $\left(10^{\circ}\right)$ postures.

\section{Statistical Analysis}

Paired 2-tailed Student t-tests were used to compare the mean value of each parameter (ADI, lateral or anteroposterior atlas dimension, and spinal canal area) between a neutral and a slightly flexed posture and between the 


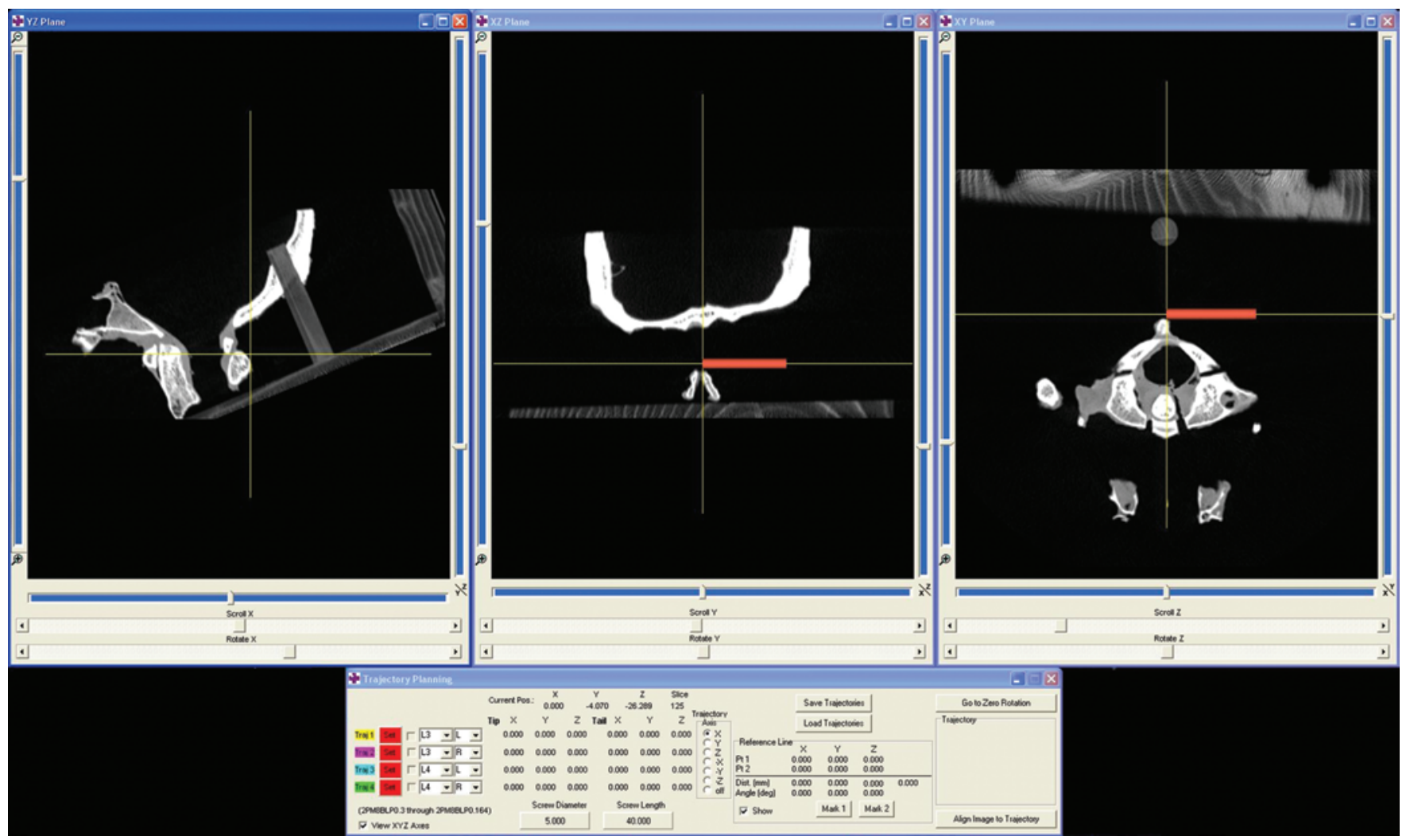

FIG. 3. Screen shot of measurements of the area of the spinal canal using 3D imaging software developed by our laboratory. The software displays 3 mutually orthogonal slices through the CT volume and allows rotating and scrolling until a true axial slice through C-2 (right window) is obtained. The image scaling factor ( $\mathrm{mm} / \mathrm{pixel}$ ) was obtained from an overlaid graphic object (red bar) of known dimension. The graphic axial slice was exported to freeware in which the spinal canal area was computed by counting pixels.

two sequences of injury. Statistical significance was established at $\mathrm{p}<0.05$.

\section{Results}

Both anteroposterior and lateral atlantal dimensions increased by $14.8 \%$ and $7.8 \%$, respectively $(\mathrm{p}<0.001)$, between the intact condition and the final compounded injury (i.e. fracture with ligamentous impairment) (Fig. 4). However, there was no significant difference in either atlas dimension $(p>0.45)$ between the neutral and flexed posture for either injury sequence (Groups A and B).

From the neutral to the $10^{\circ}$ flexed posture, ADI increased by $0.2 \mathrm{~mm}(2.5 \%)$ in intact spines and by $0.8 \mathrm{~mm}$ $(6.25 \%)$ after a Jefferson fracture without TAL disruption (Group A), but the changes were not significant ( $p>0.6$, Fig. 5). However, when the TAL was impaired (TAL disrupted, Group B) or disrupted with fracture (final condition), ADI increased by $0.4 \mathrm{~mm}(34 \%)$ in Group B and by $0.42 \mathrm{~mm}(25 \%)$ in the final condition, between the neutral and the flexed conditions. The difference was statistically significant $(\mathrm{p}<0.005)$ in both cases. There were no differences between the specimens in terms of which side of the TAL was cut $(\mathrm{p}=0.64)$.

In the intact condition and the condition with fracture and TAL impairment (final condition), there were no changes in spinal canal area between the neutral and flexed postures (Fig. 6). The area of the spinal canal increased by $2.2 \%$ during flexion after TAL impairment and decreased by $3.5 \%$ during flexion, but the differences were not significant $(\mathrm{p}>0.70)$.

\section{Discussion}

Treatment of atlas fractures is based on whether the fractures occur in isolation or in combination with other cervical spine injuries and on the integrity of the TAL, which is best assessed using high-resolution MRI. ${ }^{3}$ Isolated atlas fractures without injury to the TAL or those associated with bony avulsion of the medial tubercle should be followed for instability with flexion-extension radiography. Typically, the need for surgical fixation is determined by ligamentous impairment concomitant with the bony fracture. ${ }^{9}$

Spence et al. studied the axial load of lateral mass displacement required to disrupt the TAL in 10 human specimens. ${ }^{20}$ They suggested that, if the sum of the displacement of lateral masses of the atlas exceeded $6.9 \mathrm{~mm}$ with respect to the $\mathrm{C}-2$ lateral edges in the presence of an atlas burst fracture, the TAL could plausibly be torn. Their suggestion was based on the assumption that an intact TAL would not be associated with lateral mass spread. Soon after Spence et al. published their findings, medical professionals applied this so-called "rule of Spence" to plain 


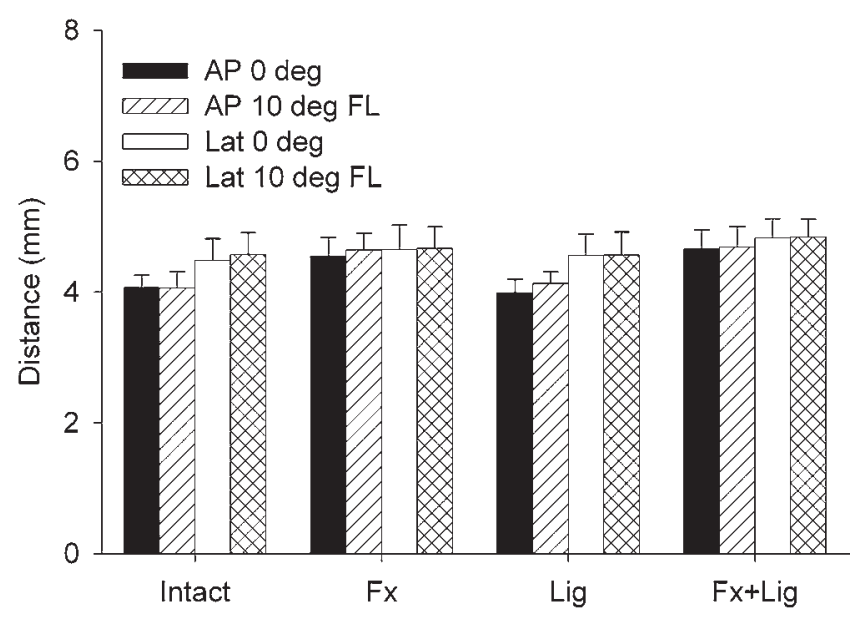

FIG. 4. Mean anteroposterior (AP) and lateral diameters of the atlas in each condition (neutral and $10^{\circ}$ flexion). Error bars show standard deviation. $\mathrm{FL}=$ flexion; $\mathrm{Fx}=$ fracture; $\mathrm{Lig}=$ ligament.

transoral radiographs to diagnose a ruptured TAL. However, radiographs do not directly visualize the TAL.

Dickman et al. ${ }^{2}$ reported that applying the rule of Spence to diagnose a TAL injury after an atlas fracture would result in missing $61 \%$ of cases-only $39 \%$ of their cases exceeded the $6.9-\mathrm{mm}$ lateral spread criteria. Other authors have postulated that the ADI as measured on flexion radiographs is the best diagnostic tool for assessing the integrity of the TAL..$^{15}$ Dickman et al. ${ }^{2}$ also studied the accuracy of the measurement of the ADI for the diagnosis of TAL impairment. Their data showed that using the accepted $\leq 3 \mathrm{~mm}$ ADI to represent normal anatomy resulted in as many as $26 \%$ of patients' injuries being judged as normal despite the presence of a TAL injury. The authors suggested that a slightly flexed lateral plain radiograph would provide a more accurate method for indirect diagnosis of a TAL injury. ${ }^{2}$

Since 1996, research, including Part 1 of this study, ${ }^{18}$ reflects the controversy surrounding the identification and treatment of atlantoaxial fractures. . $, 6,-11,14,19,21,22$ Kakarla et al. advised that the integrity of the TAL after injury should be assessed by CT and MRI to ensure diagnosis of this

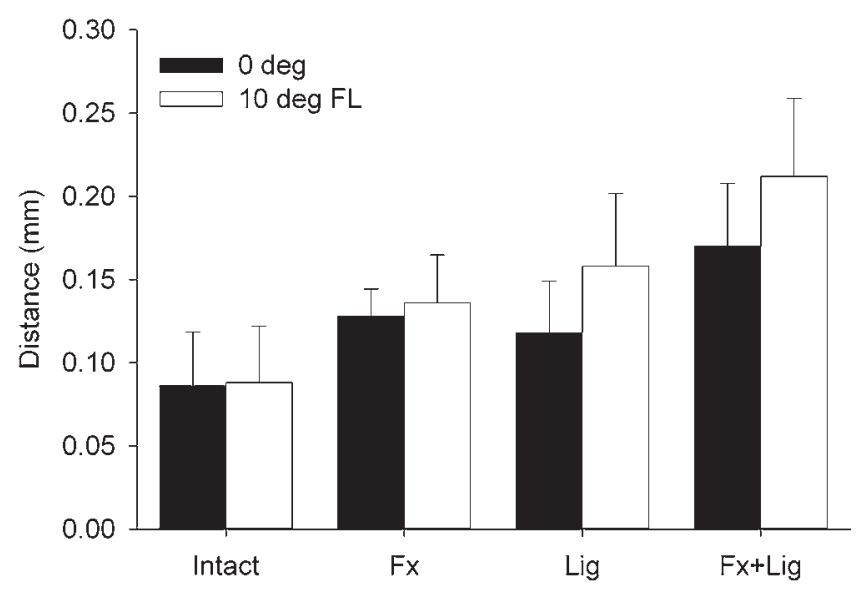

FIG. 5. Mean ADI in each condition demonstrating how it was affected by flexion. Error bars show standard deviation.

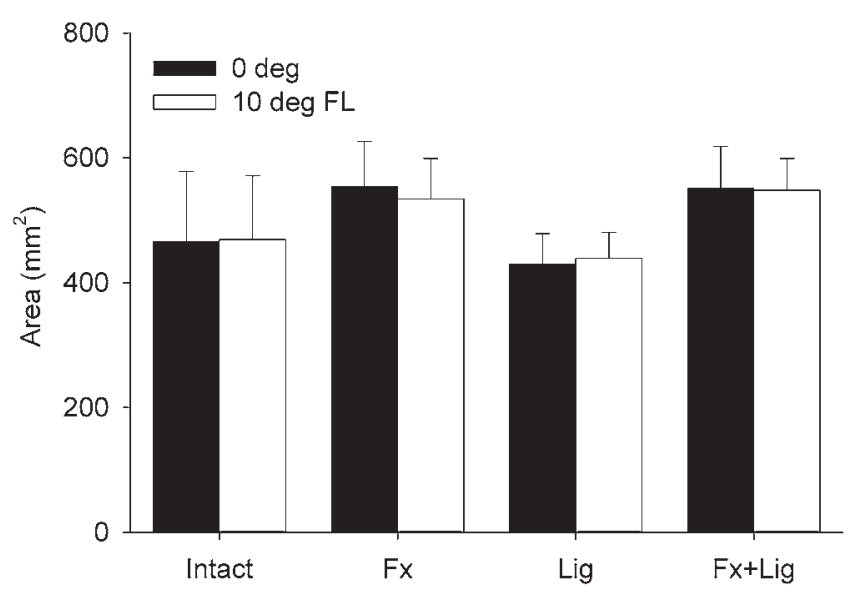

FIG. 6. Mean spinal canal area in neutral and flexed positions, demonstrating lack of change with flexion. Error bars show standard deviation.

potentially devastating condition. ${ }^{9}$ Advances in MRI, visualization techniques, and protocols have played a major role in assessment of the TAL and related membranes in the upper cervical spine. ${ }^{1,4,11,12}$

Whether CT or MRI approaches are of equal or differing utility for identifying CVJ injuries is still debated. Nonetheless, MRI has become the gold standard for the assessment of the CVJ-ligamentous complex. In an emergency department setting, however, CT with multiplanar reconstruction is the most affordable and accepted means of evaluating acute CVJ trauma. ${ }^{5}$ Furthermore, access to CT is almost twice that of access to MRI, with $9.41 \mathrm{CT}$ scanners compared with 5.68 MRI scanners per 1,000,000 population..$^{24}$ Therefore, we focused our efforts on developing a new method to assess TAL impairment based on CT alone.

Our results indicate that the change in the ADI between a neutral and a $10^{\circ}$ flexed position is a much more accurate way of assessing TAL impairment than previous methods. The ADI increased dramatically (almost 5-fold) from neutral to flexed in specimens with ligamentous impairment. In contrast, there were no significant changes when the TAL was intact, even in this highly unstable model, without paraspinal muscle action. Although the diameter of the atlas increased significantly across all conditions, there were no significant changes between neutral and flexed positions.

On the basis of these data, we propose a new diagnostic criterion to assess impairment of TAL on CT. On a cervical supine CT scan, an increase of more than $15 \%$ in the ADI between a neutral position (without head support) and a slightly flexed position (with a pillow or head holder) should be considered highly suspicious for a TAL injury. Although an angle of $10^{\circ}$ was selected for this study, the exact target angle for optimal recognition of TAL injury is unknown. An angle change of $10^{\circ}$ may be impractical in patients who have been placed in a cervical collar; further study would therefore be needed to test whether a reliable diagnosis using this method can be achieved with whatever angle change is allowed by the collar.

Our cadaveric model study did not represent the axial compressive forces generated by the cervical musculature. 
Therefore, the atlantal lateral mass spread might have been smaller than would occur in a trauma patient. However, the specimens were compressed under physiological parameters, and paraspinal musculature was dissected to create a highly unstable model. Panjabi et al. used an average of $2860 \mathrm{~N}$ to produce an immediate CVJ injury and performed their measurements thereafter. ${ }^{16}$ Their results would be difficult to extrapolate to a clinical setting, because when patients are evaluated the CVJ is only under the compressive force generated by the cervical musculature and the force of gravity. Nevertheless, our study represents the worst-case scenario: a Jefferson fracture associated with complete disruption of the TAL with no soft tissue present to support the bony structure.

This method could be applied to clinical practice because the change in the area of the spinal canal is minimal. That the area of the spinal canal does not change with slight flexion indicates that careful flexion of the cervical spine should be safe even in the presence of a Jefferson's atlas fracture and complete disruption of TAL. Our findings indicate the need for a prospective clinical study to assess the clinical sensitivity and specificity of this method in an emergency department setting. Changes in the ADI between neutral and flexed positions should be compared in patients suspected of CVJ bony and/or ligamentous injuries. Future studies of TAL injury in actual patients will provide further evidence regarding the sensitivity and specificity of this method compared with the MRI method.

\section{Conclusions}

This study explored the possibility of using 2 CT scans to evaluate the integrity of the TAL. The neutral scan represents a scan as currently obtained in trauma patients. The slightly flexed scan represents a scan obtained with some support behind the patient's head to induce slight but not full flexion. The degree of flexion $\left(10^{\circ}\right)$ used was small enough to avoid encroachment of the spinal canal. Our results indicate that this method may be more sensitive than the rule of Spence for evaluating the integrity of TAL in a clinical setting. We suggest that an increase of more than $15 \%$ in the ADI on CT scans between a neutral and a slightly flexed position is highly suspicious of a TAL impairment requiring surgical stabilization. Because CT scanning is more readily available worldwide than MRI, this method could be useful across a broad clinical spectrum.

\section{References}

1. Debernardi A, D’Aliberti G, Talamonti G, Villa F, Piparo M, Collice M: The craniovertebral junction area and the role of the ligaments and membranes. Neurosurgery 68:291-301, 2011

2. Dickman CA, Greene KA, Sonntag VK: Injuries involving the transverse atlantal ligament: classification and treatment guidelines based upon experience with 39 injuries. Neurosurgery 38:44-50, 1996

3. Dickman CA, Mamourian A, Sonntag VK, Drayer BP: Magnetic resonance imaging of the transverse atlantal ligament for the evaluation of atlantoaxial instability. J Neurosurg 75:221-227, 1991

4. Farooki S, Seeger LL: Magnetic resonance imaging in the evaluation of ligament injuries. Skeletal Radiol 28:61-74, 1999

5. Gonzalez LF, Fiorella D, Crawford NR, Wallace RC, FeizErfan I, Drumm D, et al: Vertical atlantoaxial distraction injuries: radiological criteria and clinical implications. J Neurosurg Spine 1:273-280, 2004

6. Guiot B, Fessler RG: Complex atlantoaxial fractures. J Neurosurg 91 (2 Suppl):139-143, 1999

7. Hadley MN, Dickman CA, Browner CM, Sonntag VK: Acute traumatic atlas fractures: management and long term outcome. Neurosurgery 23:31-35, 1988

8. Jo KW, Park IS, Hong JT: Motion-preserving reduction and fixation of $\mathrm{C} 1$ Jefferson fracture using a C1 lateral mass screw construct. J Clin Neurosci 18:695-698, 2011

9. Kakarla UK, Chang SW, Theodore N, Sonntag VK: Atlas fractures. Neurosurgery 66 (3 Suppl):60-67, 2010

10. Kontautas E, Ambrozaitis KV, Kalesinskas RJ, Spakauskas B: Management of acute traumatic atlas fractures. J Spinal Disord Tech 18:402-405, 2005

11. Krakenes J, Kaale BR, Moen G, Nordli H, Gilhus NE, Rorvik J: MRI assessment of the alar ligaments in the late stage of whiplash injury-a study of structural abnormalities and observer agreement. Neuroradiology 44:617-624, 2002

12. Krakenes J, Kaale BR, Moen G, Nordli H, Gilhus NE, Rorvik J: MRI of the tectorial and posterior atlanto-occipital membranes in the late stage of whiplash injury. Neuroradiology 45:585-591, 2003

13. Landells CD, Van Peteghem PK: Fractures of the atlas: classification, treatment and morbidity. Spine (Phila Pa 1976) 13:450-452, 1988

14. Miller CP, Brubacher JW, Biswas D, Lawrence BD, Whang PG, Grauer JN: The incidence of noncontiguous spinal fractures and other traumatic injuries associated with cervical spine fractures: a 10-year experience at an academic medical center. Spine (Phila Pa 1976) 36:1532-1540, 2011

15. Oda T, Panjabi MM, Crisco JJ III, Oxland TR, Katz L, Nolte LP: Experimental study of atlas injuries. II. Relevance to clinical diagnosis and treatment. Spine (Phila Pa 1976) 16 (10 Suppl):S466-S473, 1991

16. Panjabi MM, Oda T, Crisco JJ III, Oxland TR, Katz L, Nolte LP: Experimental study of atlas injuries. I. Biomechanical analysis of their mechanisms and fracture patterns. Spine (Phila Pa 1976) 16 (10 Suppl):S460-S465, 1991

17. Panjabi MM, Oxland TR, Parks EH: Quantitative anatomy of cervical spine ligaments. Part I. Upper cervical spine. J Spinal Disord 4:270-276, 1991

18. Perez-Orribo L, Snyder LA, Kalb S, Elhadi AM, Hsu F, Newcomb AGUS, et al: Comparison of CT versus MRI measurements of transverse atlantal ligament integrity in craniovertebral junction injuries. Part 1: A clinical study. J Neurosurg Spine [epub ahead of print February 26, 2016. DOI: 10.3171/2015.9.SPINE13808]

19. Sanborn MR, DiLuna ML, Whitmore RG, Storm PB: Fluoroscopically guided, transoral, closed reduction, and halo vest immobilization for an atypical C-1 fracture. J Neurosurg Pediatr 7:380-382, 2011

20. Spence KF Jr, Decker S, Sell KW: Bursting atlantal fracture associated with rupture of the transverse ligament. J Bone Joint Surg Am 52:543-549, 1970

21. Tuli S, Tator CH, Fehlings MG, Mackay M: Occipital condyle fractures. Neurosurgery 41:368-377, 1997

22. Vieweg U, Meyer B, Schramm J: Differential treatment in acute upper cervical spine injuries: a critical review of a single-institution series. Surg Neurol 54:203-211, 2000

23. Vollmer DG, Eichler ME, Jenkins AL III: Assessment of the cervical spine after trauma, in Winn R (ed): Youmans Neurological Surgery, ed 6. Philadelphia: Elsevier, 2011, pp 3166-3180

24. World Health Organization, Department of Essential Health 
Technologies: Baseline Country Survey on Medical Systems. Geneva: WHO Press, 2010

25. Yamaguchi S, Eguchi K, Kiura Y, Takeda M, Kurisu K: Posterolateral protrusion of the vertebral artery over the posterior arch of the atlas: quantitative anatomical study using three-dimensional computed tomography angiography. $\mathbf{J}$ Neurosurg Spine 9:167-174, 2008

\section{Disclosures}

The authors report no conflict of interest concerning the materials or methods used in this study or the findings specified in this paper.

\section{Author Contributions}

Conception and design: Crawford, Perez-Orribo, Kalb, Hsu, Theodore. Acquisition of data: Perez-Orribo, Kalb, Snyder, Malhotra. Analysis and interpretation of data: Crawford, Perez-Orribo. Drafting the article: Kalb, Snyder, Malhotra. Critically revising the article: Crawford. Reviewed submitted version of manuscript: Crawford, Perez-Orribo, Elhadi. Statistical analysis: Crawford, Perez-Orribo. Administrative/technical/material support: Lefevre, Elhadi, Newcomb. Study supervision: Crawford, Theodore.

\section{Supplemental Information Companion Papers}

Perez-Orribo L, Snyder LA, Kalb S, Elhadi AM, Hsu F, Newcomb AGUS, et al: Comparison of CT versus MRI measurements of transverse atlantal ligament integrity in craniovertebral junction injuries. Part 1: A clinical study. DOI: 10.3171/2015.9.SPINE13808.

\section{Correspondence}

Neil R. Crawford, c/o Neuroscience Publications, Barrow Neurological Institute, St. Joseph's Hospital and Medical Center, 350 W. Thomas Rd., Phoenix, AZ 85013.email: neuropub@ dignityhealth.org. 